\title{
Surface-Chemistry-Mediated Control of Individual Magnetic Helical Microswimmers in a Swarm
}

\section{Journal Article}

\section{Author(s):}

Wang, Xiaopu; Hu, Chengzhi; Schurz, Lukas; de Marco, Carmela; Chen, Xiangzhong; Pané, Salvador; Nelson, Bradley (1)

Publication date:

2018-06-26

Permanent link:

https://doi.org/10.3929/ethz-b-000273463

Rights / license:

In Copyright - Non-Commercial Use Permitted

Originally published in:

ACS Nano 12(6), https://doi.org/10.1021/acsnano.8b02907

Funding acknowledgement:

702128 - 3D-printed magnetic microfluidics for applications in life sciences (EC)

743217 - Soft Micro Robotics (EC) 


\section{Surface Chemistry-Mediated Control of Individual Magnetic Helical Microswimmers in a Swarm}

Xiaopu Wang, Chengzhi Hu*, Lukas Schurz, Carmela De Marco, Xiangzhong Chen, Salvador

Pané, and Bradley J. Nelson*

Institute of Robotics and Intelligent Systems, ETH Zurich, Tannenstrasse 3, CH-8092 Zurich, Switzerland

Keywords: magnetic helical microswimmers, selective control, swarm control, surface functionalization, wettability

ABSTRACT: Magnetic helical microswimmers, also known as artificial bacterial flagella (ABFs), perform 3D navigation in various liquids under low-strength rotating magnetic fields by converting rotational motion to translational motion. ABFs have been widely studied as carriers for targeted delivery and release of drugs and cells. For in vivo/in vitro therapeutic applications, control over individual groups of swimmers within a swarm is necessary for several biomedical applications such as drug delivery or small-scale surgery. In this work, we present the selective control of individual swimmers in a swarm of geometrically and magnetically identical ABFs by modifying their surface chemistry. We confirm experimentally and analytically that the forward/rotational velocity ratio of $A B F s$ is independent of their surface coatings when the swimmers are operated 
below their step-out frequency (the frequency requiring the entire available magnetic torque to maintain synchronous rotation). We also show that ABFs with hydrophobic surfaces exhibit larger step-out frequencies and higher maximum forward velocities, compared to their hydrophilic counterparts. Thus, selective control of a group of swimmers within a swarm of ABFs can be achieved by operating the selected ABFs at a frequency that is below their step-out frequencies but higher than the step-out frequencies of unselected ABFs. The feasibility of this method is investigated in water and in biologically relevant solutions. Selective control is also demonstrated inside a Y-shaped microfluidic channel. Our results present a systematic approach for realizing selective control within a swarm of magnetic helical microswimmers. 
Magnetic micro- and nanorobots are tetherless miniaturized devices capable of swimming in liquid by means of rotating, oscillating magnetic fields or magnetic field gradients. ${ }^{1-4}$ These small-scale devices have shown great potential in biomedical applications such as diagnostics, ${ }^{5,6}$ targeted drug delivery, ${ }^{7-9}$ minimally invasive surgery, or detoxification. ${ }^{10-12}$ Additionally, some of these micro- and nanorobots have been recently tested in vivo. ${ }^{13,14}$ However, many aspects must be further addressed to achieve clinical translation of micro- and nanorobots. A major hurdle is the independent control of multiple magnetic swimmers. While magnetic manipulation of swarms has been successfully demonstrated ${ }^{13}$ selective control over a reduced group of swimmers within a team requires further investigation. Selective control of small-scale robots provides more precision in certain tasks such as targeted drug delivery or surgery at smallscales. ${ }^{15-17}$ Sitti and co-workers demonstrated that motion differentiation can be achieved using microrobots with distinct geometric and magnetic features. ${ }^{18,19}$ Capelleri and co-workers have also reported the independent actuation of multiple magnetic microobjects by means of an electromagnetic setup consisting of 64 microcoils that can generate local magnetic fields. ${ }^{20}$ More recently, Rahmer et al. have shown that independent control on helical magnetic micromachines can be achieved by a combination of rotating magnetic fields and gradients, ${ }^{21}$ where rotating magnetic fields are used for their corkscrew locomotion. The gradients are employed to lock the micromachines except for those placed in specific small volumes. To date, research on independent control of microobjects has focused on varying the magnetic fields.${ }^{18-22}$ However, these strategies become challenging as the size of the objects become smaller. Alternatively, one can exploit physicochemical interactions between the surface of mobile small-scale machines and their swimming environment to achieve independent motion. 
In this paper, we explore how surface wettability affects the swimming behavior of magnetic helical microswimmers, also known as artificial bacterial flagella (ABFs), in DI water and biologically relevant aqueous media, and how surface chemistry can be exploited to achieve an independent control on microswimmers. It has been reported that hydrophobic surfaces can significantly reduce drag. ${ }^{23-28}$ To this end, identical magnetic helical microswimmers are fabricated and their surfaces are subsequently functionalized with different chemistries to change their wettability properties. We experimentally find a non-linear relationship between the stepout frequency and the contact angle, which is also verified analytically. We conclude that this approach can be used to selectively manipulate individual helical microswimmers by modulating the frequency of azimuthal magnetic fields.

\section{RESULTS AND DISCUSSION}

Fabrication of ABFs and the Surface Modification. Helical microswimmers were fabricated using two-photon polymerization of photosensitive polymers as reported earlier. ${ }^{29,30}$ Each batch consisted of more than 10000 devices. The polymeric microhelices were subsequently coated with nickel for magnetic actuation, and then with gold. The gold layer served to prevent the nickel from oxidation and to facilitate the surface functionalization with thiol chemistry. Figure 1a shows an SEM micrograph of an array of magnetic microhelices. To modify their surface wettability, the microswimmers were functionalized with thiol- and thioether-based compounds terminated with different moieties such as hydroxyl-, benzyl- and fluorine-containing groups. We have chosen thiol and thioether functionalization due to the robustness of the sulfur-gold (Au-S) interaction. $^{31,32}$ To ensure successful functionalization, the cleanliness of the gold surface is crucial. For this reason, the functionalization of the helical microswimmers was realized by 
immersing the structures for $10 \mathrm{~min}$ in isopropanol (IPA) containing the functional compounds. An illustration of the sequential coating of the helical microswimmers is shown in Figure $1 \mathrm{~b}$.

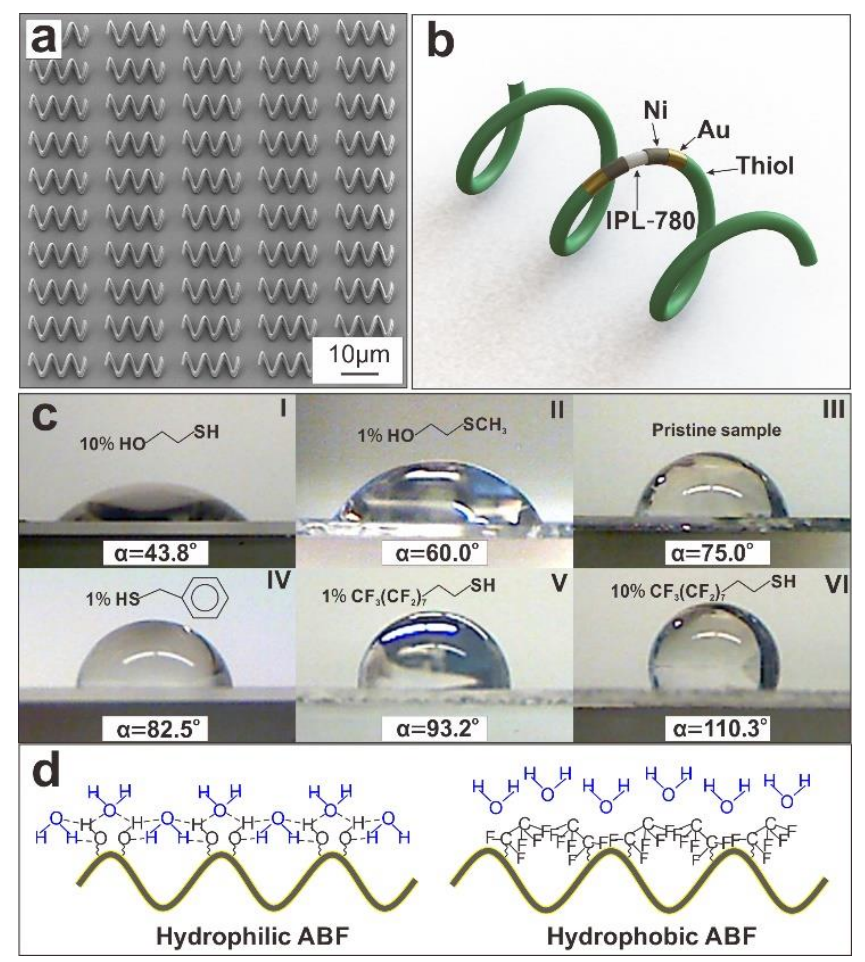

Figure 1. (a) An SEM image of an array of helical microswimmers on a glass substrate. (b) Schematic showing the sequential coating of a helical microswimmer; (c) Water contact angles of different substrates treated with thiol- or thioether-based molecules; the roman number at the top right of each picture corresponds to a different surface functionalization (see Table 1); (d) Illustration of the interaction between water and ABFs with different surface wettability.

The water contact angle values for different treated substrates were used to characterize the wettability of surface-modified helical swimmers. Table 1 summarizes the details of the functional compounds tested and the resulting water contact angles of the treated substrates. The contact angle values shown in Table 1 correspond to the average of 6 measurements. Water contact angle images corresponding to each type of surface-modified microswimmers are shown 
in Figure 1c. As expected surfaces modified with compounds containing hydroxyl moieties (Table 1, I and II) exhibit the lowest values of contact angle, thus revealing a hydrophilic nature. In contrast, those surfaces functionalized with trifluoromethyl moieties (Table 1, V and VI) show the highest hydrophobicity, which is characterized by higher contact angles.

Compared to hydrophilic surfaces, hydrophobic surfaces exhibit lower friction ${ }^{25}$ in water because of the slippage between the surface and water. As shown in Figure 1d, when the surface contains hydrophilic moieties such as hydroxyls, hydrogen bonds are established between the surface compounds and water molecules. However, for hydrophobic moieties such as trifluoromethyls, the intermolecular forces between the surface and water molecules are weak. In this case, one can expect higher slippage hydrophobic surfaces and lower friction.

Table1. The water contact angle of the substrates containing ABFs with different surface modifications.

\begin{tabular}{lll}
\hline Type & Materials & Water contact angles $\left(^{\circ}\right)$ \\
\hline I & $10 \% \mathrm{v} / \mathrm{v} \mathrm{HSCH} \mathrm{CH}_{2} \mathrm{OH}$ in IPA & $43.8 \pm 1.5$ \\
II & $1 \% \mathrm{v} / \mathrm{v} \mathrm{CH} \mathrm{SCH}_{2} \mathrm{CH}_{2} \mathrm{OH}$ in IPA & $60.0 \pm 0.4$ \\
III & Pristine sample & $75.0 \pm 0.1$ \\
& & $82.5 \pm 0.1$ \\
IV & $1 \% \mathrm{v} / \mathrm{v} \mathrm{C}_{6} \mathrm{H}_{5} \mathrm{CH}_{2} \mathrm{CH}_{2} \mathrm{SH}$ in IPA & \\
& & \\
$\mathrm{V}$ & $1 \% \mathrm{v} / \mathrm{v} \mathrm{CF}_{3}\left(\mathrm{CF}_{2}\right)_{7} \mathrm{CH}_{2} \mathrm{CH}_{2} \mathrm{SH}$ in IPA & $93.2 \pm 0.6$ \\
\hline
\end{tabular}




VI $\quad 10 \% \mathrm{v} / \mathrm{v} \mathrm{CF}_{3}\left(\mathrm{CF}_{2}\right)_{7} \mathrm{CH}_{2} \mathrm{CH}_{2} \mathrm{SH}$ in IPA $110.3 \pm 2.3$

Swimming Performance of ABFs in a Low Reynolds Liquid. When ABFs swim in a fluid, a resistive force from the fluid is experienced by each ABF. Normally, at a certain magnetic rotating field, the magnetic torque offsets the resistive torque, and ABFs swim steadily with a constant velocity where the rotating velocity synchronizes with the rotational frequency of the magnetic field. However, as the rotational speed of external magnetic field increases, a maximum magnetic torque $\left(T_{m}{ }^{\max }\right)$ of the $\mathrm{ABF}$ will be reached. In this circumstance, the magnetization of the microswimmer is perpendicular to the external magnetic field. The rotational frequency at this moment is called the step-out frequency ( $\left.\omega_{\text {step-out }}\right)$. When increasing the rotational frequency further, the drag torque on the ABF increases and becomes higher than $T_{m}{ }^{\max }$, thus causing a torque unbalance on the ABF. Consequently, both forward and rotational velocities of $\mathrm{ABF}$ decrease. At this point, forward and rotational velocities reach their maxima at the rotational step-out frequency $\omega_{\text {step-out. }}$

When a helix swims in a fluid, the general relationship between external force $(F)$, external torques $(T)$, forward velocities $(v)$ and rotational velocities $(\omega)$ of helical objects can be represented by the propulsion matrix..$^{33,34}$

$\left[\begin{array}{l}F \\ T\end{array}\right]=\left[\begin{array}{ll}a & b \\ b & c\end{array}\right]\left[\begin{array}{l}v \\ \omega\end{array}\right]$

where, $a, b$, and $c$ are the elements of the propulsion matrix, which are mainly determined by the geometry and surface properties of the swimmer, as well as fluid properties. From Equation 1, it 
can be found that when ABFs are manipulated under a rotating magnetic field, the magnetic force is 0 and only magnetic torque is applied. When ABFs have identical geometry and volume of magnetic material, the maximum magnetic torque that can be applied by an external magnetic field to helical swimmers $\left(T_{m}{ }^{\max }\right)$ is a constant. In this case, based on the propulsion matrix, the relationship between rotating frequency and forward swimming speed, and the relationship between step out frequency ( $\left.\omega_{\text {step-out }}\right)$ and $T_{m}^{\max }$ can be obtained as,

$\frac{v}{\omega}=-\frac{b}{a}$

$\omega_{\text {step-out }}=\frac{a}{a c-b^{2}} T_{m}^{\max }$

Experimentally, we studied the swimming performance of different types of ABFs in deionized water with a rotating magnetic field of $1 \mathrm{mT}$ and $3 \mathrm{mT}$. To clarify the numbering of ABFs from different types of chemical modification, $\mathrm{ABF}(\mathrm{N})$ is used to denote $\mathrm{ABF}$ of type $\mathrm{N}(e . g$. I/II/III/IV/V/VI). The forward swimming velocity of each ABF against the rotational frequency of the magnetic field at $3 \mathrm{mT}$ is shown in Figure 2a. Each data point represents an average of nine trials characterized from three different ABFs with the same coating (three trials for each ABF). As shown in Figure 2a, the forward swimming speeds of ABFs increase linearly against the rotating frequency of the magnetic field and then reach the peak at the step-out frequency. When the rotating frequency of the external magnetic field is higher than ABFs' step-out frequency, the swimming speed of the ABFs decrease dramatically. Meanwhile, the slopes of forward velocity against frequency (i.e. forward/rotational velocity rate) of differentially coated ABFs are the same when they are operated beneath their step-out frequencies. Further, differentially coated 
ABFs exhibit different step-out frequencies. It is also clear from the data in Figure $2 b$ that the step-out frequency is non-linearly dependent on the contact angle.
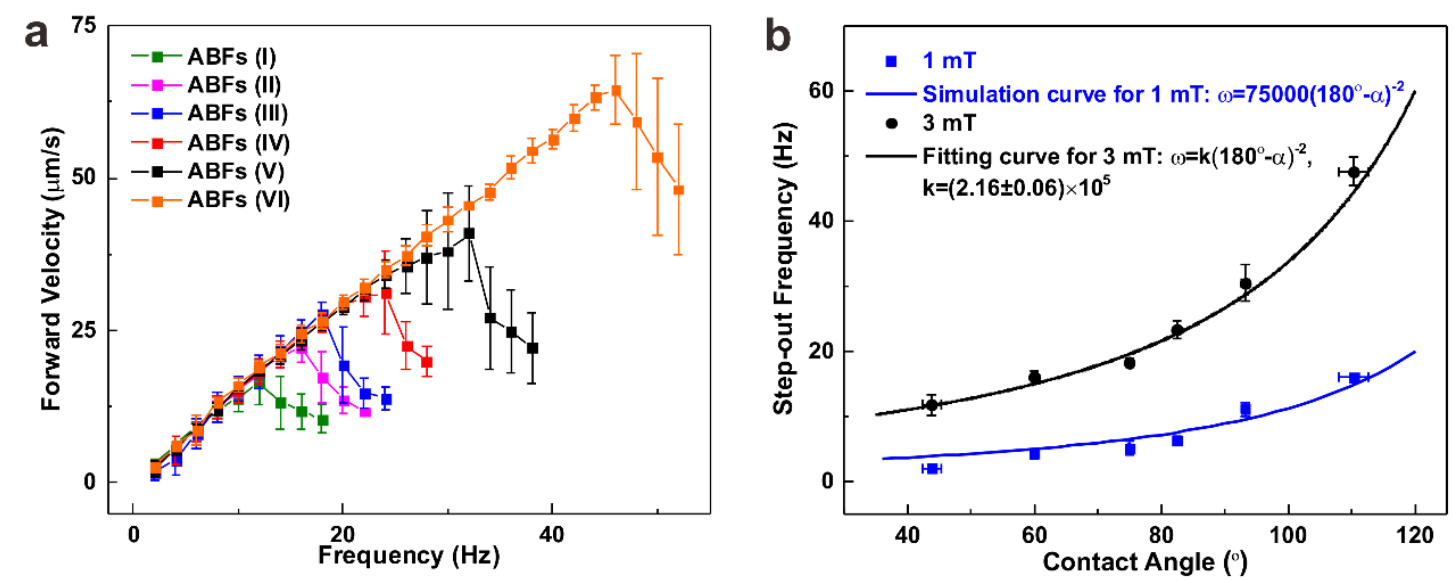

Figure 2. The wirelessly controlled swimming performance of ABFs in water. (a) The forward swimming velocities of different $\mathrm{ABF}$ as a function of the applied rotating frequency of magnetic fields at $3 \mathrm{mT}$. (b) The relationship between contact angle of each type of ABFs and step-out frequency at $1 \mathrm{mT}$ and $3 \mathrm{mT}$, respectively.

To understand these results, a comprehensive analytical model that considers the surface wettability is employed. Lighthill's work ${ }^{35}$ shows that the water drag force $(d f)$ applied on a small region of a helical swimmer with a length of ds is given as, $d f=\xi v_{s} d s$, where $v_{s}$ is the velocity, and $\xi$ is the local resistance coefficient in no-slip boundary condition. For tangential and normal directions, the values of local resistance coefficients are different (the numerical expression is shown in supporting information). Considering surface wettability of ABFs, a noslip boundary condition is not suitable. Slippage, which is characterized using slip length $(g)^{36}$ 
must be considered. Assuming $Q$ is the coefficient that denotes the contribution of interfacial slippage, it can be used to correct the resistance coefficient. Hence, the drag force becomes $d f=$ $Q \xi v_{s} d s$. Following Bocquet and Barrat's argument, in which the drag force is inversely proportional to the slip length, ${ }^{37,38}$ the relationship of $Q$ and g should be $Q \propto 1 / \mathrm{g}$. Moreover, the slip length is a function of surface water contact angle $(\alpha): g(\alpha) \propto\left(180^{\circ}-\alpha\right)^{-2} .{ }^{39}$ Thus, $Q=$ $q\left(180^{\circ}-\alpha\right)^{2}$, where $q$ is a constant. By analyzing the force at the slip boundary condition according to resistive force theory, the values of each element of the propulsion matrix were obtained (the derivation details can be found in the supporting information).

$$
\begin{aligned}
& a=2 n \pi R\left(\frac{\xi_{\|} \cos ^{2}(\theta)+\xi_{\perp} \sin ^{2}(\theta)}{\sin (\theta)}\right) q\left(180^{\circ}-\alpha\right)^{2} \\
& b=2 n \pi R^{2}\left(\xi_{\|}-\xi_{\perp}\right) \cos \theta q\left(180^{\circ}-\alpha\right)^{2} \\
& c=2 n \pi R^{3}\left(\frac{\xi_{\|} \sin ^{2}(\theta)+\xi_{\perp} \cos ^{2}(\theta)}{\sin (\theta)}\right) q\left(180^{\circ}-\alpha\right)^{2}
\end{aligned}
$$

In Equation 4-6, $n$ is the turns of the $\mathrm{ABF}$, and $R$ and $\theta$ are the radius of each turn and the pitch angle (the angle between an ABF's long axis and the tangent at each point along the helix structure), respectively. The local tangential resistance coefficient and normal resistance coefficient are $\xi_{\|}$and $\xi_{\perp}$, respectively, which can be determined by the viscosity of liquid and geometry parameters of ABFs, as given by Lighthill. ${ }^{35}$

Substituting Equation 4-6 into Equation 2-3

$$
\frac{v}{\omega}=-\frac{b}{a}=-R \frac{\left(\xi_{\|}-\xi_{\perp}\right) \cos \theta \sin (\theta)}{\xi_{\|} \cos ^{2}(\theta)+\xi_{\perp} \sin ^{2}(\theta)}
$$


$\omega_{\text {step-out }}=\frac{a}{a c-b^{2}} T_{m}^{\max }=\frac{\left(\xi_{\|} \cos ^{2} \theta+\xi_{\perp} \sin ^{2} \theta\right) \sin \theta}{2 \pi n R^{3} \xi_{\|} \xi_{\perp}\left(\cos ^{2} \theta+\sin ^{2} \theta\right)^{2} q\left(180^{\circ}-\alpha\right)^{2}} T_{m}^{\max }$

Thus, the forward/rotational velocity rate $\frac{v}{\omega}$, is not a function of water contact angle $\alpha$, which agrees well with our experimental results. Moreover, $T_{m}{ }^{m a x}$ is a constant at a certain magnetic field, hence the step-out frequency is $\omega_{\text {step-out }} \propto\left(180^{\circ}-\alpha\right)^{-2}$, which also matches well with our experimental results. Using $\omega_{\text {step-out }} \propto\left(180^{\circ}-\alpha\right)^{-2}$, the experimental values of step-out frequency at $3 \mathrm{mT}$ as a function of contact angles were fitted with a power function curve. The fitting curve is shown as the black curve in Figure 2b. Experimentally, it is confirmed that the relationship between step-out frequency and water contact angle is,

$\omega_{\text {step-out }}=k\left(180^{\circ}-\alpha\right)^{-2}, k=(2.16 \pm 0.06) \times 10^{5}$

By substituting Equation 9 and the value of each parameter into Equation 8, the value of $q$ (0.0644) was obtained (the derivation details can be found in supporting information). Thus, the coefficient that denotes the contribution of interfacial slippage has been used to correct the resistance coefficient, which is: $Q=0.0644\left(180^{\circ}-\alpha\right)^{2}$.

$T_{m}{ }^{\max }$ depends linearly on the applied magnetic field ${ }^{1}$, thus, as can be observed in Equation 8 , $\omega_{\text {step-out }}$ is also a linear function of the applied magnetic field. Based on the theory above and the obtained $Q$ value, a relationship between step-out frequency and contact angle at a specific magnetic field can be obtained. For a magnetic field of $1 \mathrm{mT}, \omega_{\text {step-out }(1 \mathrm{mT})}=75000\left(180^{\circ}-\alpha\right)^{-2}$. The red curve in Figure $2 \mathrm{~b}$ represents the simulation results of $1 \mathrm{mT}$. Experimental results realized at $1 \mathrm{mT}$ also confirmed the relationship between step-out frequency and water contact angle. We conclude that our analytical model and the coefficient $(Q)$ proposed are representative of $\mathrm{ABF}$ performance. In summary, we confirmed that the swimming behavior of geometrically 
and magnetically identical ABFs is the same when they are manipulated below their step-out frequency. Depending on their surface wettability, the step-out frequency of the swimmers varies and increases with the contact angle. The more hydrophobic the surface of the swimmer, the higher its step-out frequency. Based on these results, selective control of individual ABFs in a swarm can be achieved.

Selective Control of a Swarm of ABFs in deionized water. We experimentally verified selective control of four helical swimmers that are geometrically and magnetically identical but with distinct surface chemistry (Figure 3 and Video S1). The four ABFs used here are ABF(I), $\mathrm{ABF}(\mathrm{II}), \mathrm{ABF}(\mathrm{III})$, and $\mathrm{ABF}(\mathrm{VI})$, respectively. Their corresponding step-out frequencies can be found in Figure 2a. As illustrated in Figure 3a, applying an external rotating magnetic field with a given strength, four $\mathrm{ABFs}$ were aligned along the same direction when the rotating frequency of the external magnetic field $(\omega)$ was set to 0 . At a frequency of $\omega 1$, which is not higher than ABF(I)'s step-out frequency, all ABFs swam with the same forward speed. When increasing the rotating frequency of the external magnetic field to a frequency $\omega 2$, which is higher than $\mathrm{ABF}(\mathrm{I})$ 's step-out frequency but not higher than $\mathrm{ABF}(\mathrm{II})$ 's step-out frequency, $\mathrm{ABF}(\mathrm{I})$ stepped out and its forward velocity decreased, while the other ABFs kept swimming at a higher and equal forward velocity. Similarly, when increasing the rotating frequency of the rotating magnetic field to a value $\omega 3$, which is higher than $\mathrm{ABF}(\mathrm{II})$ 's step-out frequency but not higher than $\mathrm{ABF}(\mathrm{III})$ 's step-out frequency, both $\mathrm{ABF}(\mathrm{I})$ and $\mathrm{ABF}(\mathrm{II})$ stepped out, while $\mathrm{ABF}(\mathrm{III})$ and $\mathrm{ABF}(\mathrm{VI})$ kept swimming faster at the same forward velocity. Finally, when the rotating frequency was increased to $\omega 4$, which is higher than $\mathrm{ABF}(\mathrm{III})$ 's step-out frequency but not higher than $\mathrm{ABF}(\mathrm{VI})$ 's step-out frequency, $\mathrm{ABF}(\mathrm{I}), \mathrm{ABF}(\mathrm{II})$ and $\mathrm{ABF}(\mathrm{III})$ stepped out and only 
$\mathrm{ABF}(\mathrm{VI})$ swam at a higher forward velocity. The experimental sequences shown in Figure $3 \mathrm{~b}-\mathrm{e}$ represent the situation of $\omega 1, \omega 2, \omega 3$, and $\omega 4$, respectively.

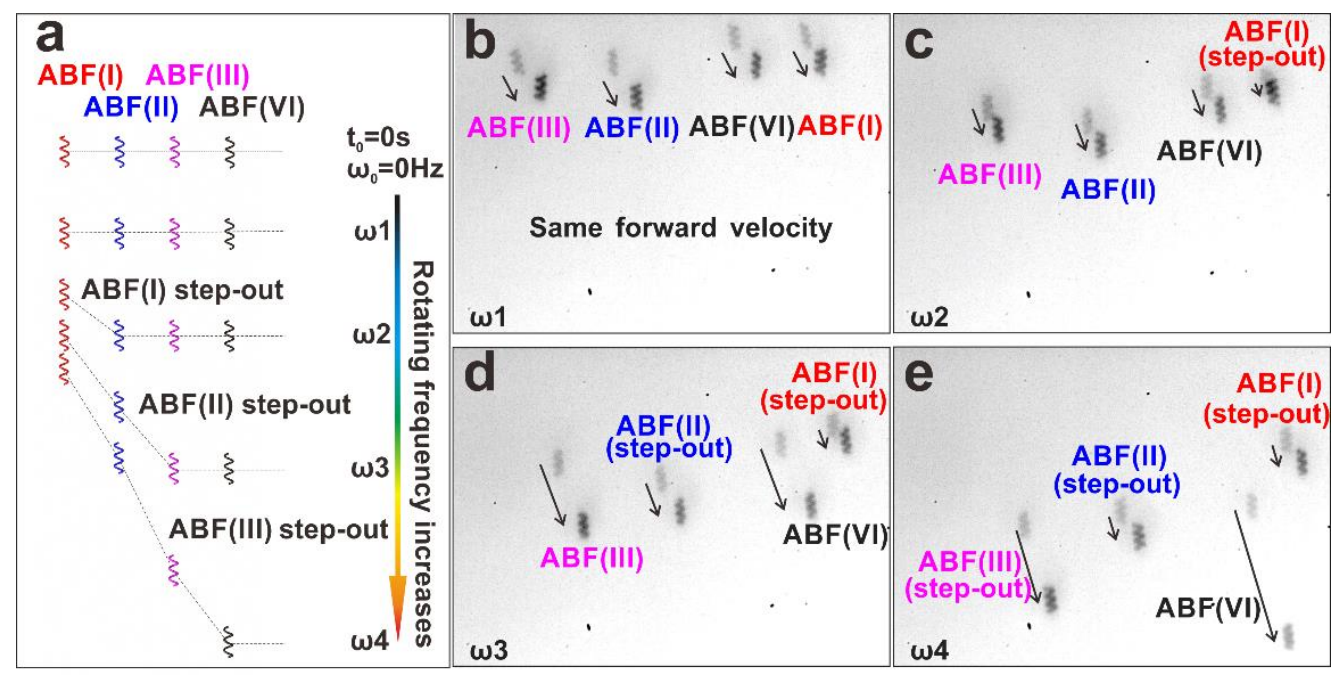

Figure 3. Four differentially coated ABFs swim in deionized water at $3 \mathrm{mT}$. (a) Illustration of selective control to differentially coated ABFs, (b-e) Screenshots of Video S1 showing the movement of four differentially coated ABFs. The arrows indicate ABFs' displacement when applying the corresponding frequency of rotating magnetic field.

To demonstrate selective control of ABFs within a swarm, $\mathrm{ABFs}(\mathrm{III})$ and $\mathrm{ABFs}(\mathrm{VI})$ were manipulated in a Y-shaped microchannel, as shown in Figure 4 (Video S2). In this experiment, a magnetic field of $1 \mathrm{mT}$ and a rotating frequency of $8 \mathrm{~Hz}$ was initially employed. As shown in Figure $4 b$, the two groups of ABFs swam in the microchannel indistinguishably. By increasing the rotating frequency to $16 \mathrm{~Hz}, \mathrm{ABFs}$ with hydrophilic surfaces stepped out and their swimming velocity decreased. In contrast, the hydrophobic group of ABFs swam at a higher forward velocity. At this point, if the direction of the rotating magnetic field is changed towards the upper left branch (as shown in Figure 4c), the hydrophobic group continues swimming until they disappear from view. Afterwards, the magnetic field was increased to $2 \mathrm{mT}$, resulting in an 
increase in the step-out frequency of the hydrophilic ABFs (which is higher than $16 \mathrm{~Hz}$ ), and the direction was changed towards the bottom left branch. The hydrophilic group of ABFs moved along the lower branch towards another destination (as shown in Figure 4d). Hence, we demonstrate that distinctly coated ABFs can swim together into a channel with the same speed (Figure 4b). By reaching the step-out frequency of a group of swimmers it is possible to lock or decrease the speed of a group of swimmers, while increasing the forward velocity of another group. In this way, one can efficiently separate a group of swimmers and guide them to different destinations. (Figure 4c and Figure 4d). Similar results were found when controlling smaller ABFs (13 ABFs in the field of view) with the same strategy (Video S3).

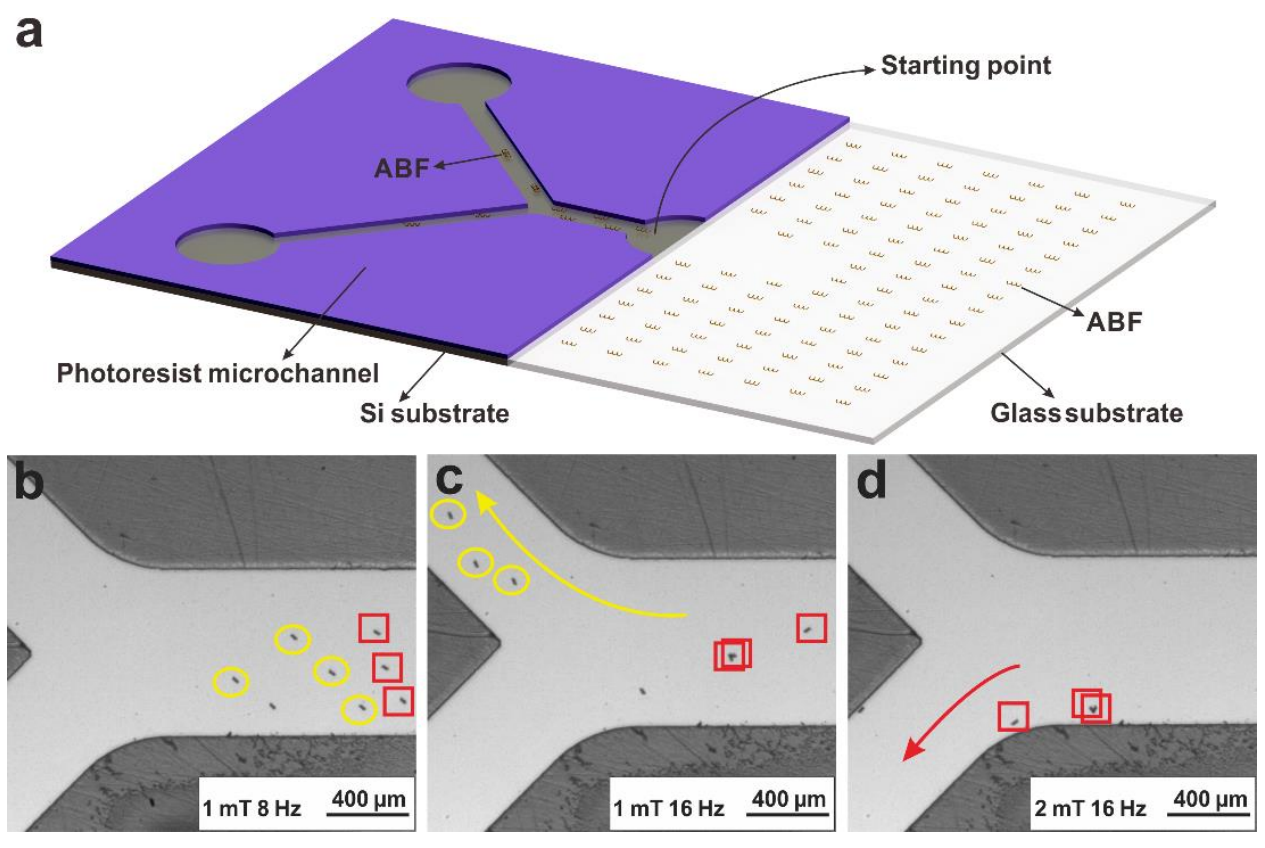

Figure 4. A swarm of differentially coated ABFs swims to different branches of a Y-shaped microchannel in deionized water. (a) The illustration of ABFs that are transferred to and swim on the silicon substrate of a Y-shaped microchannel. (b) All ABFs enter the main channel at the same speed. Those marked with yellow rings are $\mathrm{ABFs}(\mathrm{VI})$, and those marked red squares are 
$\mathrm{ABFs}(\mathrm{III})$. (c) A group of ABFs goes into the upper left branch and others remain in the main channel. (d) The remaining ABFs go into the bottom left branch.

Our approach may find applications where ABFs can precisely deliver drugs to different targets or to the same target at different times with distinct therapeutic agents. During the application of the proposed method, many parameters can influence the contact angle, such as the surface chemistry of the helical microswimmer which has been confirmed in this paper, the roughness of helical microswimmer, and the properties of liquid. The contact angle can also be influenced if the helical microswimmer has been altered due to the contact with the liquid, such as a chemical reaction, or absorption. Thus, there are many ways to tune the contact angle of helical microswimmers. Among them, surface chemistry modification provides an inexpensive, easy, reliable approach to differentiate the contact angle of helical microswimmers within a widely distributed range. Until now, many approaches for surface chemistry modification have been developed ${ }^{40-44}$. The surface chemistry modification used in this work is based on sulfur-gold interface and thoils with different functional groups, which has previously been widely employed in the fields of chemistry, physics, molecular biology, pharmaceutical engineering and materials science, but not in microrobotics ${ }^{45-47}$. The covalent bond between gold and sulfur gives rise to a robust and tunable surface chemistry modification. Thus, it is feasible to realize selective control of helical microswimmers in different solutions (such as water and aqueous solutions that mimic a physiological environment) by surface chemistry modification, as long as the resulting contact angles are different.

Selective Control of a Swarm of ABFs in a biologically relevant solution. When small-scale objects are deployed in biological fluids, biomolecules such as proteins, carbohydrates and other 
components interact with the object's surface, which could affect their swimming performance. Therefore, we evaluated the locomotion of differentially coated ABFs in a biologically relevant phosphate-buffered saline (PBS, $\mathrm{pH}=7.4$ ) solution containing $38 \mathrm{mg} / \mathrm{ml}$ of bovine serum albumin (BSA) and $0.9 \mathrm{mg} / \mathrm{ml}$ of glucose. $\mathrm{ABFs}(\mathrm{III})$ and $\mathrm{ABFs}(\mathrm{VI})$ were tested with an applied rotating magnetic field of $1 \mathrm{mT}$. The curves of forward velocity as a function of the rotating magnetic field frequency for these two types of ABFs are shown in Figure 5a. As expected, the step-out frequency of $\mathrm{ABF}(\mathrm{VI})$ is higher than that of $\mathrm{ABF}(\mathrm{III})$. As shown in Figure $5 \mathrm{~b}, 5 \mathrm{c}$ and Video $\mathrm{S} 4$, by applying a frequency of $8 \mathrm{~Hz}$, which is lower than the step-out frequency of both, two different ABFs exhibited the same forward velocity. When applying a frequency of $18 \mathrm{~Hz}$, which is higher than $\mathrm{ABF}(\mathrm{III})$ 's step-out frequency and smaller than $\mathrm{ABF}(\mathrm{VI})$ 's step-out frequency, $\mathrm{ABF}(\mathrm{VI})$ speeded up showing a corkscrew locomotion, while, $\mathrm{ABF}(\mathrm{III})$ slowed down exhibiting a combination of wobbling locomotion and surface walker behavior. We then went on testing in the same solution the swimming behavior of a swarm consisting of $\mathrm{ABFs}(\mathrm{III})$ and $\mathrm{ABFs}(\mathrm{VI})$, as shown in Figure 5d, 5e and Video S5 (about 20 ABFs within the field of view). When a frequency of $8 \mathrm{~Hz}$ was applied, all ABFs exhibited the same motion. For a frequency of $16 \mathrm{~Hz}$, a group of ABFs (No.3, No.8, No.10, and No.12, marked with red ovals in Figure 5e) slowed down, while other ABFs speeded up. The experiments showed that our approach also works in complex aqueous environments. 


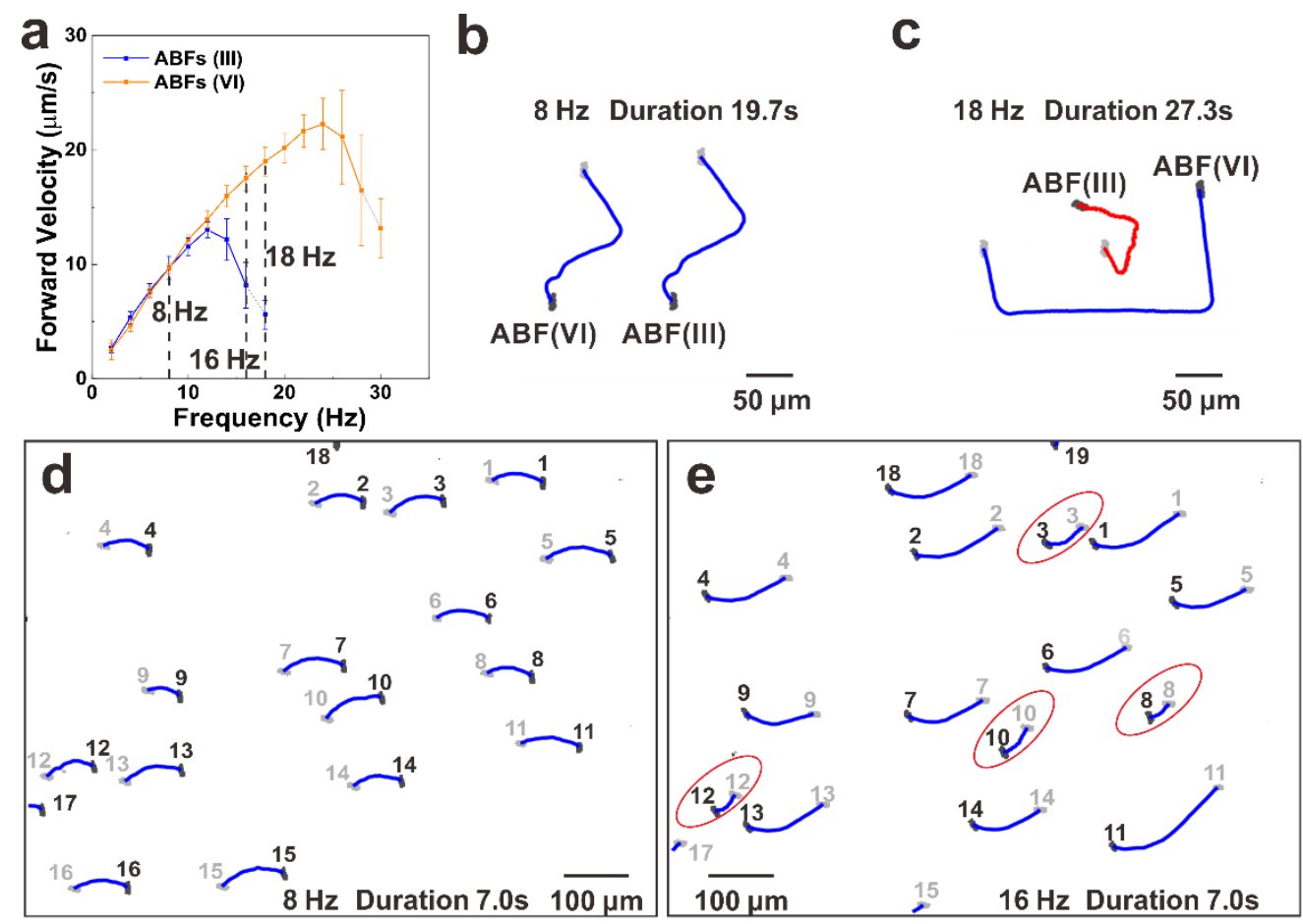

Figure 5. Motion of $\mathrm{ABFs}(\mathrm{III})$ and $\mathrm{ABFs}(\mathrm{VI})$ in BSA-Glucose-PBS solution. (a) Velocity analysis of two types of ABFs at $1 \mathrm{mT}(\mathrm{n}=3)$. (b) Tracking of one $\mathrm{ABF}(\mathrm{III})$ and one $\mathrm{ABF}(\mathrm{VI})$ at 1mT 8Hz. (c) Tracking of one $\mathrm{ABF}(\mathrm{III})$ and one $\mathrm{ABF}(\mathrm{VI})$ at $1 \mathrm{mT} 18 \mathrm{~Hz}$. (d) Tracking of a swarm consisting of $\mathrm{ABFs}(\mathrm{III})$ and $\mathrm{ABFs}(\mathrm{VI})$ at $1 \mathrm{mT} 8 \mathrm{~Hz}$. (e) Tracking of a swarm consisting of $\mathrm{ABFs}(\mathrm{III})$ and $\mathrm{ABFs}(\mathrm{VI})$ at $1 \mathrm{mT} 16 \mathrm{~Hz}$ (about $20 \mathrm{ABFs}$ in the field of view).

\section{CONCLUSION}

This work investigates the swimming behavior of ABFs with different hydrophilic and hydrophobic surface coatings in water at room temperature. The effect of surface wettability on the swimming behavior of ABFs is studied. It is confirmed experimentally and theoretically that all ABFs with different surface wettability exhibit the same behavior when manipulated below their step-out frequencies. ABFs reach their step-out frequency and slow down sequentially as the rotating frequency increases. The step-out frequency and maximum forward velocity increase 
as the surface property changes from hydrophilic to hydrophobic, and a nonlinear relationship between step-out frequency and contact angle caused by the interfacial slippage is observed. A slip boundary condition is applied to incorporate interfacial slippage into a helical swimmer swimming model. A helical microswimmer's swimming at a slip boundary condition is characterized by the universal coefficient $(Q)$, which denotes the contribution of interfacial slippage to correct the resistance coefficients. This work analyzes the basic principles of ABF swimming in liquids with a slip boundary condition. It also provides a method for selectively controlling a team of ABFs within a swarm. We also experimentally demonstrate that one can efficiently separate a group of swimmers and guide them to different destinations exploiting differences in their wettability. Our approach works not only in deionized water, but also in biologically relevant aqueous environments. Modifying ABF surface chemistry is a promising approach for the selective control of swimmers within a swarm, and holds great promise for targeted drug delivery and microsurgery applications. Furthermore, the method can be complementary to other existing strategies for selective control.

\section{EXPERIMENTAL SECTION}

Fabrication and Surface Modification of ABFs. The ABFs were fabricated using a 3D Direct Laser lithography (Nanoscribe $\mathrm{GmbH}$ ) on a glass substrate. IPL-780 was used as the photoresist. After writing, the helical structures were developed in IPA for $10 \mathrm{~min}$, and then dried with a nitrogen gun. The fabricated helical structures were coated with Ni and Au by electron beam evaporator (Plassys-II MEB550SL). During this process, a rotational speed of $20 \mathrm{rpm}$ and a tilt of $15^{\circ}$ were applied to the rotational stage to achieve a homogeneous coating on the $\mathrm{ABF}$ surface. Unless otherwise specified, all ABFs have the length of $15 \mu \mathrm{m}$, the diameter of each turn 
of $5 \mu \mathrm{m}$, and the metal coating layers of $50 \mathrm{~nm} \mathrm{Ni}$ with $5 \mathrm{~nm} \mathrm{Au}$ on the top. For the test in $\mathrm{Y}$ shaped channel, bigger ABFs were also used for a better visibility. The length of them was 30 $\mu \mathrm{m}$, the diameter of each turn was $10 \mu \mathrm{m}$, and the metal coating layers were $90 \mathrm{~nm} \mathrm{Ni}$ with $9 \mathrm{~nm}$ $\mathrm{Au}$ on the top.

To obtain ABFs with different surface wettability, a series of thiols/thioethers with different hydrophilic/hydrophobic moieties were used to modify their surfaces. The thiol/thioether IPA solutions include $10 \% \mathrm{v} / \mathrm{v} 2$-mercaptoethanol in IPA, $1 \%$ v/v 2-(methylthio) ethanol in IPA, $1 \%$ v/v 2-phenylethanethiol in IPA, $1 \% \mathrm{v} / \mathrm{v} 1 \mathrm{H}, 1 \mathrm{H}, 2 \mathrm{H}, 2 \mathrm{H}$-perfluorodecanethiol in IPA, and 10\% v/v $1 \mathrm{H}, 1 \mathrm{H}, 2 \mathrm{H}, 2 \mathrm{H}$-perfluorodecanethiol in IPA. The surface modification of ABFs was conducted by treating the pre-fabricated $\mathrm{ABFs}$ samples with $\mathrm{O}_{2}$ plasma for 1 min and then immersing the plasma treated ABFs into each solution for 10 min, followed by rinsing with IPA, and then drying with a nitrogen gun. All chemical reagents used in this work were of analytical reagent grade and were used as received from Sigma-Aldrich without further purification. Deionized water was adopted to prepare all aqueous environments.

Characterization of Wettability. The wettability of each type of ABFs' surface was characterized by measuring the water contact angle of the corresponding thiol/thioether coated substrates at room temperature. Three points were chosen on each substrate for measuring the contact angle. A digital camera and the sample wafers were placed horizontally and at the same height, and $2 \mu \mathrm{L}$ water was dripped onto the selective position and then the photo was taken immediately to avoid water evaporation. The photos of water droplets were used to characterize the contact angles. 
Swimming Test. The swimming tests were performed in a Helmholtz setup made by three pairs of orthogonal coils as shown in Figure S3. The sample and a silicon substrate were placed near each other under water inside the sample holder, and the sample holder was placed at the center of the setup to make sure the uniformity of the magnetic field. A microprobe (T-4-22, GGB Industries, INC.) was used to transport $\mathrm{ABFs}$ from glass substrate onto the silicon substrate under water. Three ABFs with a same surface modification were used for characterizing the swimming properties of each type of $\mathrm{ABFs}$, and each $\mathrm{ABF}$ was tested three times. The forward/rotational velocity rate, step-out frequency, and maximum forward velocity were analyzed.

Fabrication of Microchannel. A positive photoresist AZ 40XT was used to fabricate the microchannel by photolithography. By spinning coating AZ 40XT onto a clean silicon wafer at $1750 \mathrm{rpm}$ for 20 seconds, a $40 \mu \mathrm{m} \mathrm{AZ} \mathrm{40XT} \mathrm{layer} \mathrm{was} \mathrm{achieved.} \mathrm{The} \mathrm{AZ} \mathrm{40XT} \mathrm{coated} \mathrm{wafer}$ was soft baked at $126^{\circ} \mathrm{C}$ for $7 \mathrm{~min}$, and exposed at h-line of $420 \mathrm{~mJ} / \mathrm{cm}^{2}$ using Karl Süss mask aligner MA6 under a shadow mask, then post baked at $105^{\circ} \mathrm{C}$ for 100 seconds. Afterwards, by cutting the wafer using dicing saw, smaller wafers with single microchannel pattern were obtained. Finally, the patterns were developed in AZ 726MIF developer for $3 \times 60$ seconds and washed with water repeatedly to remove the exposed parts so that the microchannel was formed. The mask used and its geometry parameters are shown in supporting information and Figure S4.

Selective Control of ABFs. $A B F$ samples (ABF(I), $A B F(I I), A B F(I I I)$, and $A B F(V I))$ were immersed into deionized water in the sample holder together with a silicon substrate, and the sample holder was placed in the center of the Helmholtz setup. ABFs of these four types were transported onto the same silicon substrate in deionized water using a microprobe (T-4-22). To 
prevent the aggregation, ABFs were placed at a distance more than double of their body length. Afterwards, a rotating magnetic field of $3 \mathrm{mT}$ was applied. While changing the rotating frequency from $4 \mathrm{~Hz}$ to $24 \mathrm{~Hz}$, the swimming behaviors of these four ABFs were recorded.

A Y-shaped microchannel was designed to demonstrate the feasibility of selective control of ABFs inside a branched environment (e.g. blood vessel). The microchannel was immersed into water in the sample holder together with the $\mathrm{ABFs}$ samples $(\mathrm{ABF}(\mathrm{III})$ and $\mathrm{ABF}(\mathrm{VI}))$, and the sample holder was placed in the center of the Helmholtz setup. A microprobe (T-4-22) was used to transport ABFs to the "starting point" (as shown in Figure 4a) on the Si substrate of the microchannel. Then magnetic field was applied to manipulate ABFs.

To assess the swimming behavior of differentially coated ABFs in a biologically relevant environment, a phosphate buffer saline (PBS) solution containing $38 \mathrm{mg} / \mathrm{ml}$ of BSA and 0.9 $\mathrm{mg} / \mathrm{ml}$ of glucose was prepared. ABF samples (ABFs(III) and ABFs(VI)) were immersed together into this biologically relevant solution, and sonicated for 2 min to release two types of ABFs into the solution. The concentration of ABFs was controlled to be approximately 5000 per milliliter to prevent the aggregation. A silicon substrate was put into the sample holder and the biologically relevant solution with thousands of ABFs was transferred onto the silicon substrate with a pipette. Then the sample holder was placed in the center of the Helmholtz setup and a rotating magnetic field of $1 \mathrm{mT}$ was applied to manipulate the ABFs.

\section{ASSOCIATED CONTENT}


Supporting Information. Derivation of the values of propulsion matrix elements; calculation of the correction coefficient $(\mathrm{Q})$ in local resistance coefficient; the setup of magnetic manipulation system used for steering ABFs; fabrication of the microchannel (PDF)

Video S1: Movie showing selective control of four ABFs in water (AVI)

Video S2: Movie showing ABFs manipulated in a Y-shaped microchannel in water (bigger size $\mathrm{ABFs})(\mathrm{AVI})$

Video S3: Movie showing ABFs manipulated in a Y-shaped microchannel in water (smaller size $\mathrm{ABFs})(\mathrm{AVI})$

Video S4: Movie showing selective control of two ABFs in a biologically relevant solution (AVI)

Video S5: Movie showing selective control of a swarm of ABFs in a biologically relevant solution (AVI)

\section{AUTHOR INFORMATION}

\section{Corresponding Author}

*E-mail: huc@ethz.ch; bnelson@ethz.ch

\section{Present Addresses}


Institute of Robotics and Intelligent Systems, ETH Zurich, Tannenstrasse 3, CH-8092 Zurich, Switzerland

\section{Author Contributions}

C.H., S.P. and B.J.N. designed the research. X.W., L.S., C.D.M. and X.C. performed the research. All authors analyzed data. X.W. and C.H. wrote the paper.

\section{Funding Sources}

The work has been partially funded by the SBFI Cost Project No C16.0061 under the COST Action MP1407, and the European Research Council Advanced Grant: 743217 - Soft Micro Robotics (SOMBOT). X.W. acknowledges financial support from China Scholarship Council (No: 201504910817). C. de M. acknowledges a fellowship from the European Union's Horizon 2020 research and innovation program under the Marie Skłodowska-Curie grant agreement 702128.

\section{ACKNOWLEDGMENT}

The authors would like to thank Famin Qiu for his help during the process of ABF fabrication, and Andrew Petruska, Ayoung Hong, Erdem Can Siringil, and Samuel Charreyron for the calibration of the Helmholtz setup. The authors thank the FIRST lab of ETH Zurich for technical support.

\section{REFERENCES}


1. Peyer, K. E.; Zhang, L.; Nelson, B. J., Bio-Inspired Magnetic Swimming Microrobots for Biomedical Applications. Nanoscale 2013, 5, 1259-1272.

2. Kim, S.; Qiu, F. M.; Kim, S.; Ghanbari, A.; Moon, C.; Zhang, L.; Nelson, B. J.; Choi, H., Fabrication and Characterization of Magnetic Microrobots for Three-Dimensional Cell Culture and Targeted Transportation. Adv. Mater. 2013, 25 , 5863-5868.

3. Ghosh, A.; Fischer, P., Controlled Propulsion of Artificial Magnetic Nanostructured Propellers. Nano Lett. 2009, 9, 2243-2245.

4. Hu, C.; Aeschlimann, F.; Chatzipirpiridis, G.; Pokki, J.; Chen, X.; Puigmarti-Luis, J.; Nelson, B. J.; Pané, S., Spatiotemporally Controlled Electrodeposition of Magnetically Driven Micromachines Based on the Inverse Opal Architecture. Electrochem. Commun. 2017, 81, $97-$ 101.

5. Chałupniak, A.; Morales-Narváez, E.; Merkoçi, A., Micro and Nanomotors in Diagnostics. Adv. Drug Deliv. Rev. 2015, 95, 104-116.

6. Ergeneman, O.; Dogangil, G.; Kummer, M. P.; Abbott, J. J.; Nazeeruddin, M. K.; Nelson, B. J., A Magnetically Controlled Wireless Optical Oxygen Sensor for Intraocular Measurements. IEEE Sens. J. 2008, 8, 29-37. 
7. Qiu, F.; Mhanna, R.; Zhang, L.; Ding, Y.; Fujita, S.; Nelson, B. J., Artificial Bacterial Flagella Functionalized with Temperature-Sensitive Liposomes for Controlled Release. Sens. Actuator BChem. 2014, 196, 676-681.

8. Chen, X. Z.; Hoop, M.; Shamsudhin, N.; Huang, T.; Özkale, B.; Li, Q.; Siringil, E.; Mushtaq, F.; Di Tizio, L.; Nelson, B. J., Hybrid Magnetoelectric Nanowires for Nanorobotic Applications: Fabrication, Magnetoelectric Coupling, and Magnetically Assisted in Vitro Targeted Drug Delivery. Adv. Mater. 2016, 29, 1605458.

9. Hoop, M.; Mushtaq, F.; Hurter, C.; Chen, X.-Z.; Nelson, B.; Pané, S., A Smart Multifunctional Drug Delivery Nanoplatform for Targeting Cancer Cells. Nanoscale 2016, 8, 12723-12728.

10. Soler, L.; Magdanz, V.; Fomin, V. M.; Sanchez, S.; Schmidt, O. G., Self-Propelled Micromotors for Cleaning Polluted Water. ACS Nano 2013, 7, 9611-9620.

11. Mushtaq, F.; Guerrero, M.; Sakar, M. S.; Hoop, M.; Lindo, A. M.; Sort, J.; Chen, X.; Nelson, B. J.; Pellicer, E.; Pané, S., Magnetically Driven $\mathrm{Bi}_{2} \mathrm{O}_{3} / \mathrm{BiOCl}-\mathrm{Based}$ Hybrid Microrobots for Photocatalytic Water Remediation. J. Mater. Chem. A 2015, 3, 23670-23676.

12. Mushtaq, F.; Asani, A.; Hoop, M.; Chen, X. Z.; Ahmed, D.; Nelson, B. J.; Pané, S., Highly Efficient Coaxial $\mathrm{TiO}_{2}-\mathrm{PtPd}$ Tubular Nanomachines for Photocatalytic Water Purification with Multiple Locomotion Strategies. Adv. Funct. Mater. 2016, 26, 6995-7002. 
13. Servant, A.; Qiu, F. M.; Mazza, M.; Kostarelos, K.; Nelson, B. J., Controlled in Vivo

Swimming of a Swarm of Bacteria-Like Microrobotic Flagella. Adv. Mater. 2015, 27, 29812988.

14. Felfoul, O.; Mohammadi, M.; Taherkhani, S.; de Lanauze, D.; Xu, Y. Z.; Loghin, D.; Essa, S.; Jancik, S.; Houle, D.; Lafleur, M.; Gaboury, L.; Tabrizian, M.; Kaou, N.; Atkin, M.; Vuong, T.; Batist, G.; Beauchemin, N.; Radzioch, D.; Martel, S., Magneto-Aerotactic Bacteria Deliver Drug-Containing Nanoliposomes to Tumour Hypoxic Regions. Nat. Nanotechnol. 2016, 11, 941947.

15. Li, J. X.; Thamphiwatana, S.; Liu, W. J.; de Avila, B. E. F.; Angsantikul, P.; Sandraz, E.; Wang, J. X.; Xu, T. L.; Soto, F.; Ramez, V.; Wang, X. L.; Gao, W. W.; Zhang, L. F.; Wang, J., Enteric Micromotor Can Selectively Position and Spontaneously Propel in the Gastrointestinal Tract. ACS Nano 2016, 10, 9536-9542.

16. Li, J. X.; Angsantikul, P.; Liu, W. J.; de Avila, B. E. F.; Thamphiwatana, S.; Xu, M. L.;

Sandraz, E.; Wang, X. L.; Delezuk, J.; Gao, W. W.; Zhang, L. F.; Wang, J., Micromotors Spontaneously Neutralize Gastric Acid for Ph-Responsive Payload Release. Angew. Chem. Int. Edit. 2017, 56, 2156-2161.

17. Tottori, S.; Sugita, N.; Kometani, R.; Ishihara, S.; Mitsuishi, M., Selective Control Method for Multiple Magnetic Helical Microrobots. J. Micro-Nano Mech. 2011, 6, 89-95. 
18. Diller, E.; Giltinan, J.; Sitti, M., Independent Control of Multiple Magnetic Microrobots in Three Dimensions. Int. J. Robot. Res. 2013, 32, 614-631.

19. Diller, E.; Floyd, S.; Pawashe, C.; Sitti, M., Control of Multiple Heterogeneous Magnetic Microrobots in Two Dimensions on Nonspecialized Surfaces. IEEE T. Robot. 2012, 28, 172-182.

20. Cappelleri, D.; Efthymiou, D.; Goswami, A.; Vitoroulis, N.; Zavlanos, M., Towards Mobile Microrobot Swarms for Additive Micromanufacturing. Int. J. Adv. Robot. Syst. 2014, 11, 150.

21. Rahmer, J.; Stehning, C.; Gleich, B., Spatially Selective Remote Magnetic Actuation of Identical Helical Micromachines. Sci. Robot. 2017, 2, eaal2845.

22. Pawashe, C.; Floyd, S.; Sitti, M., Multiple Magnetic Microrobot Control Using Electrostatic Anchoring. Appl. Phys. Lett. 2009, 94, 164108.

23. Min, T.; Kim, J., Effects of Hydrophobic Surface on Skin-Friction Drag. Phys. Fluids 2004, 16, L55-L58.

24. Daniello, R. J.; Waterhouse, N. E.; Rothstein, J. P., Drag Reduction in Turbulent Flows over Superhydrophobic Surfaces. Phys. Fluids 2009, 21, 085103.

25. Ou, J.; Perot, B.; Rothstein, J. P., Laminar Drag Reduction in Microchannels Using Ultrahydrophobic Surfaces. Phys. Fluids 2004, 16, 4635-4643. 
26. Dean, B.; Bhushan, B., Shark-Skin Surfaces for Fluid-Drag Reduction in Turbulent Flow: A Review. Philos. Trans. R. Soc., A 2010, 368, 4775-4806.

27. Truesdell, R.; Mammoli, A.; Vorobieff, P.; van Swol, F.; Brinker, C. J., Drag Reduction on a Patterned Superhydrophobic Surface. Phys. Rev. Lett. 2006, 97, 044504.

28. Bhushan, B.; Jung, Y. C., Natural and Biomimetic Artificial Surfaces for Superhydrophobicity, Self-Cleaning, Low Adhesion, and Drag Reduction. Prog. Mater. Sci. 2011, 56, 1-108.

29. Qiu, F. M.; Zhang, L.; Peyer, K. E.; Casarosa, M.; Franco-Obregon, A.; Choi, H.; Nelson, B. J., Noncytotoxic Artificial Bacterial Flagella Fabricated from Biocompatible Ormocomp and Iron Coating. J. Mater. Chem. B 2014, 2, 357-362.

30. Qiu, F. M.; Fujita, S.; Mhanna, R.; Zhang, L.; Simona, B. R.; Nelson, B. J., Magnetic Helical Microswimmers Functionalized with Lipoplexes for Targeted Gene Delivery. Adv. Funct. Mater. 2015, 25, 1666-1671.

31. Love, J. C.; Estroff, L. A.; Kriebel, J. K.; Nuzzo, R. G.; Whitesides, G. M., Self-Assembled Monolayers of Thiolates on Metals as a Form of Nanotechnology. Chem. Rev. 2005, 105, 11031170. 
32. Thoden van Velzen, E. U.; Engbersen, J. F.; Reinhoudt, D. N., Self-Assembled Monolayers of Receptor Adsorbates on Gold: Preparation and Characterization. J. Am. Chem. Soc. 1994, 116, 3597-3598.

33. Purcell, E. M., Life at Low Reynolds Number. Am. J. Phys. 1977, 45, 3-11.

34. Purcell, E. M., The Efficiency of Propulsion by a Rotating Flagellum. Proc. Natl. Acad. Sci. U. S. A. 1997, 94, 11307-11311.

35. Lighthill, J., Flagellar Hydrodynamics. SIAM Rev. 1976, 18, 161-230.

36. Cottin-Bizonne, C.; Barrat, J.-L.; Bocquet, L.; Charlaix, E., Low-Friction Flows of Liquid at Nanopatterned Interfaces. Nat. Mater. 2003, 2, 237-240.

37. Barrat, J.-L.; Bocquet, L., Influence of Wetting Properties on Hydrodynamic Boundary Conditions at a Fluid/Solid Interface. Faraday Discuss. 1999, 112, 119-128.

38. Bocquet, L.; Barrat, J.-L., Hydrodynamic Boundary Conditions, Correlation Functions, and Kubo Relations for Confined Fluids. Phys. Rev. E 1994, 49, 3079.

39. Sendner, C.; Horinek, D.; Bocquet, L.; Netz, R. R., Interfacial Water at Hydrophobic and Hydrophilic Surfaces: Slip, Viscosity, and Diffusion. Langmuir 2009, 25, 10768-10781.

40. Satulu, V.; Ionita, M. D.; Vizireanu, S.; Mitu, B.; Dinescu, G., Plasma Processing with Fluorine Chemistry for Modification of Surfaces Wettability. Molecules 2016, 21, 1711. 
41. Chen, C.; Zhu, X. Y.; Gao, Q. L.; Fang, F.; Huang, X. J., Surface Modification of

Cyclomatrix Polyphosphazene Microsphere by Thiol-Ene Chemistry and Lectin Recognition. Appl. Surf. Sci. 2016, 387, 1029-1036.

42. Gao, N. N.; Zhang, Q.; Mu, Q. X.; Bai, Y. H.; Li, L. W.; Zhou, H. Y.; Butch, E. R.; Powell, T. B.; Snyder, S. E.; Jiang, G. B.; Yan, B., Steering Carbon Nanotubes to Scavenger Receptor Recognition by Nanotube Surface Chemistry Modification Partially Alleviates Nf Kappa B Activation and Reduces Its Immunotoxicity. ACS Nano 2011, 5, 4581-4591.

43. Ye, Q.; Zhou, F.; Liu, W. M., Bioinspired Catecholic Chemistry for Surface Modification. Chem. Soc. Rev. 2011, 40, 4244-4258.

44. Xue, C. H.; Guo, X. J.; Zhang, M. M.; Ma, J. Z.; Jia, S. T., Fabrication of Robust Superhydrophobic Surfaces by Modification of Chemically Roughened Fibers Via Thiol-Ene Click Chemistry. J. Mater. Chem. A 2015, 3, 21797-21804.

45. Xue, Y. R.; Li, X.; Li, H. B.; Zhang, W. K., Quantifying Thiol-Gold Interactions Towards the Efficient Strength Control. Nat. Commun. 2014, 5, 4348.

46. Hakkinen, H., The Gold-Sulfur Interface at the Nanoscale. Nat. Chem. 2012, 4, 443-455.

47. Boisselier, E.; Astruc, D., Gold Nanoparticles in Nanomedicine: Preparations, Imaging, Diagnostics, Therapies and Toxicity. Chem. Soc. Rev. 2009, 38, 1759-1782. 


\section{Table of Contents Graphic}

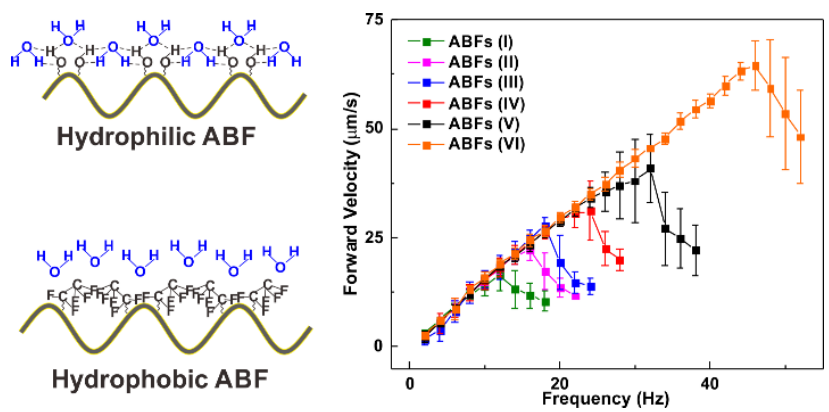

